\title{
A Comparative TEM Study of Soot Particles Derived from Used Diesel and Gasoline Engine Oils
}

\author{
Arne Janssen ${ }^{1}$, Laura Felisari ${ }^{2}$, Matthew A. Kulzick ${ }^{3}$ and M.G. Burke ${ }^{1}$ \\ ${ }^{1 .}$ Materials Performance Centre, School of Materials, The University of Manchester, Manchester, UK \\ 2. BP Technology Centre, Pangborne, Reading, UK \\ ${ }^{3 .}$ BP Corporate Research Centre, Naperville Il, USA
}

Soot formation is an undesirable effect of incomplete combustion of fuel in internal combustion engines, which amongst other factors implies an incorrect air/fuel ratio and an improper combustion temperature. Whilst most of the formed soot will be oxidized in the combustion chamber or released to the environment with the exhaust gases, a small proportion is transferred from the combustion chamber to the lubricant oil. These particles are responsible for engine wear and their formation is related to oil performance. These have been known in diesel engines but are becoming increasingly important in newer high performance gasoline engines. The aim of this project is to study the evolution of the structure and morphology of different gasoline and diesel soot samples extracted from used engine oils.

Samples for the TEM characterization were prepared after the method of La Rocca [1]. The soot containing used engine oil was first cleaned using heptane and then followed by 6 stages of centrifugation. After each stage the top phase fluid was removed and equally replaced by pure heptane. TEM investigations were conducted in a FEI Talos F200X TEM with Super X (4 Si drift detectors (SDDs)) operated at $200 \mathrm{keV}$. Conventional bright-field TEM imaging has been used to determine particle shape, size and distribution. High resolution TEM (HRTEM) was used to study the structure of single soot particles (i.e. inner core and outer shell). The chemical composition of the inorganic compounds was analyzed by STEM-XEDS.

STEM-XEDS spectrum imaging and analyses of the inorganic compounds in the gasoline and diesel sample revealed similar varieties of calcium carbonates, silicates, phosphates, and elemental sulphur with a broad particle size distribution ranging from $\mathrm{nm}$ to $\mu \mathrm{m}$. However, the inorganic compounds in the diesel soot were less concentrated and in average smaller in size.

Conventional TEM observation indicates that structure, morphology, size of primary soot particles and the degree of agglomeration are strongly influenced by the fuel type. Diesel soot particles are mainly distributed as long chained agglomerates (Figure 1a). Particle size measurements of primary diesel soot particles revealed an average diameter of $24 \mathrm{~nm}$, with a standard deviation of $6 \mathrm{~nm}$. HRTEM analyses confirmed a high degree of graphitization of the soot particles resulting in a graphitic shell with an average shell thickness of $6 \mathrm{~nm}$ and a turbostratic core (Figure 1b-c).

The analyzed gasoline soot is mainly distributed as single particles and occasionally agglomerated to globular type clusters with $<10$ of primary particles (2a). Particle size analyses for the gasoline soot revealed an average diameter of $20 \mathrm{~nm}$ with standard deviation of $4 \mathrm{~nm}$. HRTEM investigations indicate that most of the soot particles are amorphous (2b). A certain degree of graphitization was only observed on a minor amount of the soot (2c). Further characterization of soot particles derived from different gasoline and diesel engines are in progress [2]. 


\section{References:}

[1] La Rocca et al, Tribology International 16 (2013), p. 80.

[2] The authors would like to acknowledge the funding and technical support from BP through the BP International Centre for Advanced Materials (BP-ICAM) which made this research possible.
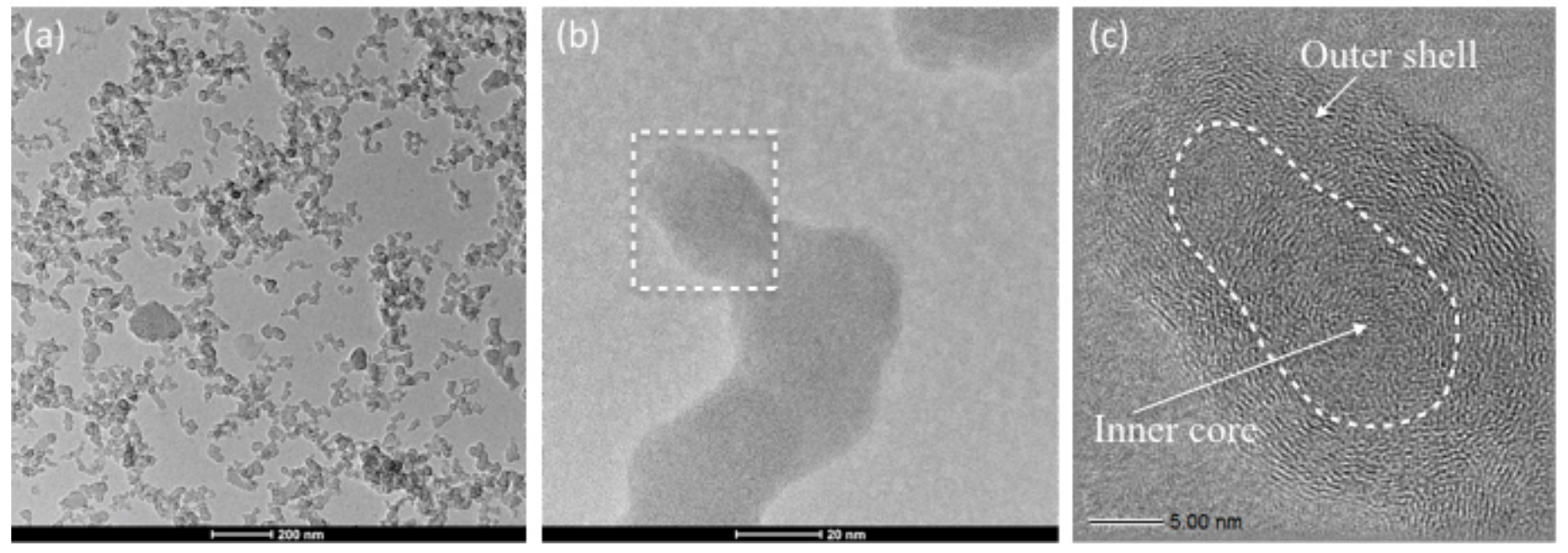

Figure 1. TEM overview image of diesel soot particles (a). Chain of agglomerated soot particles (b). (c) Higher resolution TEM image of a single diesel soot particle shown in (b) by dashed square. White dashed line in (c) highlights the layered typed outer shell and the highly disordered inner core.
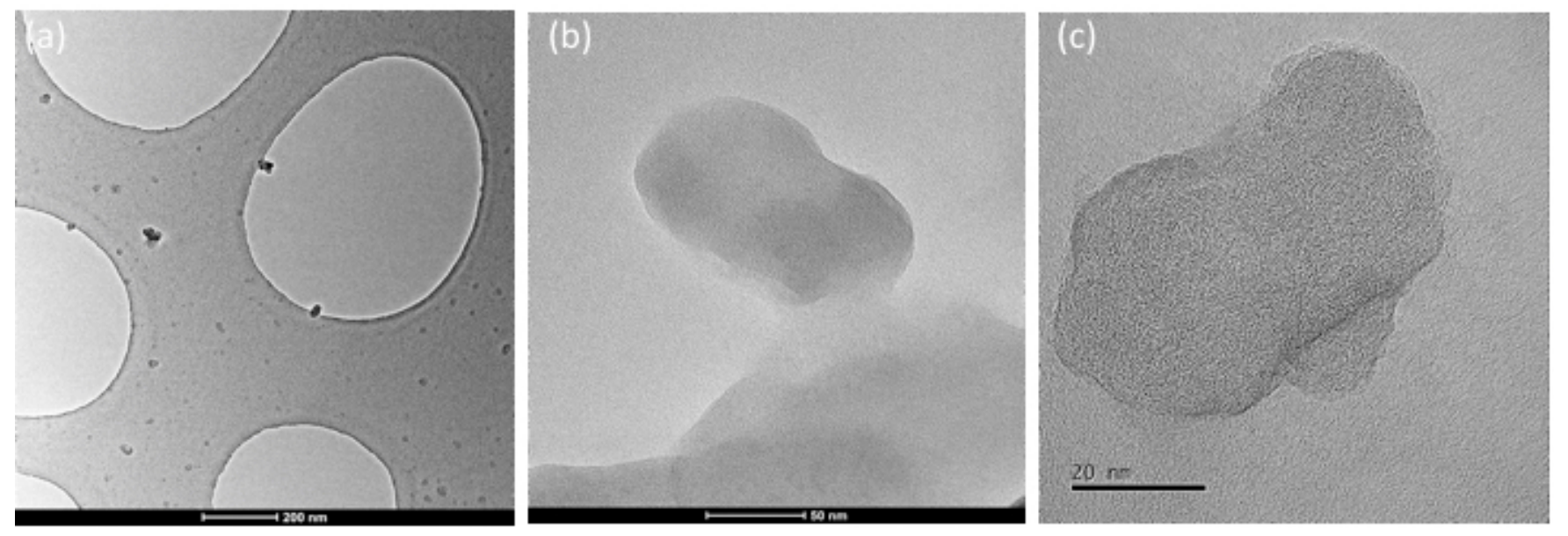

Figure 2. TEM overview image of gasoline particles (a). Amorphous gasoline soot particle (b). Minor amounts of the observed soot showed a turbostratic structure without a graphitic shell (c). 